\title{
Microbial diversity in grape musts from Austrian and South African grape varieties and regions
}

\author{
B. Bagheri ${ }^{1}$, C. Philipp ${ }^{2}$, M. Horacek ${ }^{3}$, F.F. Bauer ${ }^{1}$, and M.E. Setati ${ }^{1}$ \\ ${ }^{1}$ Department of Viticulture and Oenology, Institute for Wine Biotechnology, Stellenbosch University, Stellenbosch, South Africa \\ ${ }^{2}$ Höhere Bundeslehranstalt und Bundesamt für Wein- und Obstbau, Wienerstraße 74, 3400 Klosterneuburg, Austria \\ ${ }^{3}$ Department of Lithospheric Research, University Vienna, Althanstr. 14, 1090 Vienna, Austria \\ \# further affiliation: Lehr- und Forschungszentrum Francisco Josephinum, Rottenhauserstraße 1, 3250 Wieselburg, Austria
}

\begin{abstract}
Vitis vinifera is one of the most widely planted crops and holds important economic value in South Africa and Austria. Grapes obtained from this plant harbour a complex fungal community which plays a crucial role in the wine fermentation process and influences wine flavour and aroma. For many years the contributions of the natural yeasts has been eclipsed by the use of active dry yeast (ADY) inoculant, mainly of the species Saccharomyces cerevisiae. However, recent studies show a growing interest in deciphering the natural microbial diversity and in promoting its persistence during fermentation in order to enhance wine typicity. The current preliminary study aims to provide a first broad assessment of the fungal community fingerprint of different grape varietals from different wine producing areas in Austria and South Africa through Automated Ribosomal Intergenic Spacer Analysis (ARISA). The ARISA profiles separated the samples according to country of origin, and suggested some regional and varietal separation within each country. Future work will evaluate the contribution of these fungal communities to wine chemical composition and sensorial distinctness.
\end{abstract}

\section{Introduction}

Global wine production in 2017 was estimated at 247 million hectolitres (mhl), of which South Africa (SA) and Austria contributed 10.8 and $2.4 \mathrm{mhl}$, respectively. The two countries are ranked number 8 and 19 in global wine production [1]. South African wine production accounts for approximately $1.5 \%$ (more than 36 billion ZAR) of the National Gross Domestic Product (GDP) [2,3] whereas Austrian wine industry accounts for $1.3 \%$ (around 3.6 billion Euro) of GDP [4]. In spite of their contribution in the GPD, both countries face strong competition from the leading wine producing countries located in Europe and the Southern hemisphere such as Spain, France, Chile, Australia and New Zealand [2-4]. In order to remain competitive in the global market, it is crucial to produce wines with a unique aromatic profile that reflect the 'terroir' from which they originate. Previous studies showed that grape microbiota exhibit regional patterns ('terroir') which contribute significantly to the quality of wine $[5,6]$. However, most studies have evaluated the grape associated microbiota in different regions/ vineyards of the same country whereas the comparative analysis of grape microbiota cultivated in different vineyards of two different countries has not been undertaken previously.

Furthermore, it is unclear to what extent grape microbiota contributes to the geographical delineation of wines. Thus, the current study aims at investigating the grape associated microbiota in different vineyards of South Africa and Austria.

\section{Material and methods}

\subsection{Sampling procedure}

The current study compared fungal community structures on different wine grape cultivars in South Africa and Austria. Five Vitis vinifera (V1-V5) were obtained from three regions in Austria (AT1-AT3) during the 2017 harvest, while in South Africa two cultivars (V6 \& V7) were collected from three locations in South Africa (SA1SA3) in 2018. The grapes were destemmed and crushed in the cellar. Fifty-millilitre samples were withdrawn from the fresh must and analysed for fungal diversity and chemical composition of the must. Triplicate samples were collected and analysed for each cultivar.

\subsection{Chemical analysis}

The primary chemical parameters of the musts were analysed using the FOSS Grape Scan 2000 (Rhine Ruhr, Denmark). Glucose, Fructose, volatile acidity, tartaric acid as well as the $\mathrm{pH}$ of the juice were measured.

\subsection{Fungal community fingerprinting}

For the community analysis, total genomic DNA was extracted from the fifty millilitre grape juice samples. The samples were centrifuged at $6000 \mathrm{~g}$ for $5 \mathrm{~min}$. The cell pellet was resuspended in $5 \mathrm{~mL}$ of a buffer solution containing $0.15 \mathrm{M} \mathrm{NaCl}, 0.1 \mathrm{M}$ EDTA and $2 \%(\mathrm{w} / \mathrm{v})$ Polyvinyl pyrolidone) [7]. Two hundred microlitres of the cell suspension was used for DNA extraction. The DNA 
was extracted using the FavorPrep ${ }^{\mathrm{TM}}$ soil DNA Isolation Mini kit (Favorgen, Biotech Corp). The concentrations of extracted DNA were determined spectrophotometrically, using the NanoDrop $囚 N D-1000$ (NanoDrop Technologies Inc., Wilmington, DE, United States). Polymerase Chain reaction was performed using carboxy-flourescein labelled ITS1 primer together with ITS4 primer [8]. The PCR amplicons were separated by capillary electrophoresis using an Applied Biosystems $3130 \times l$ Genetic Analyzer (Applied Biosystems, Forster City, CA, USA) and preprocessed with Gene Mapper 4.0 software (Applied Biosystems, Forster City, CA, USA). Peaks were detected from $50 \mathrm{bp}$ to $1000 \mathrm{bp}$ based on the LIZ1200 molecular standard, however, peaks smaller than $300 \mathrm{bp}$ were discarded as they did not correspond to any known species.

\subsection{Statistical analysis}

Peaks were aligned using T-align and data was transformed to a matrix file with peak sizes and peak abundance using the Terminal Restriction enzyme Fragment Length Polymorphism (T-RFLP) Expedited (T-REX) software (http://trex.biohpc.org/) [9]. Diversity analyses were performed using Paleontological Statistics (PAST) version 3.0 [10]. Analysis of similarities (ANOSIM) based on 999 permutations together with a permutational multivariate analysis of variance (PERMANOVA) based on the Bray-Curtis distance matrices [10] was used to assess the differences in fungal communities between the Austria and South Africa, and between the grape cultivars.

\section{Results and discussion}

The current study aimed to unveil the fungal community fingerprint of different grape varietals from different wine producing areas in Austria and South Africa and evaluate the degree of similarities across the regions and varietals. Significant differences were observed in fungal community of grape musts obtained from different vineyards in South Africa and Austria (Fig. 1). Furthermore, differences in fungal community profiles were observed within the same grape variety collected from different vineyards in Austria (Fig. 2). However, irrespective of the grape variety, some unique OTUs were only detected in each vineyard. This observation suggests that each vineyard has fungal species which are the potential fungal signature of that region. Our result is in agreement with previous studies [5,6], who have suggested that Grape-associated microbial biogeography is non-randomly associated with regional and varietal across multiscale viticultural zones. The unique OTUs observed in fungal communities of each region and each grape varietal in SA and AST is particularly important since these unexplored fungal species may hold some potential for further application in local wine industries. Recent studies suggest that regional-specific "microbialterroir" significantly contributes to the quality of wine $[5,12]$, and there is a growing interest in exploring the natural microbial diversity and promoting its persistence in fermentation in order to enhance wine typicity.

In conclusion, the current preliminary study for the first time investigated the community profile of fungal species in different vineyards of South Africa and Austria. The results present a first step in the identification

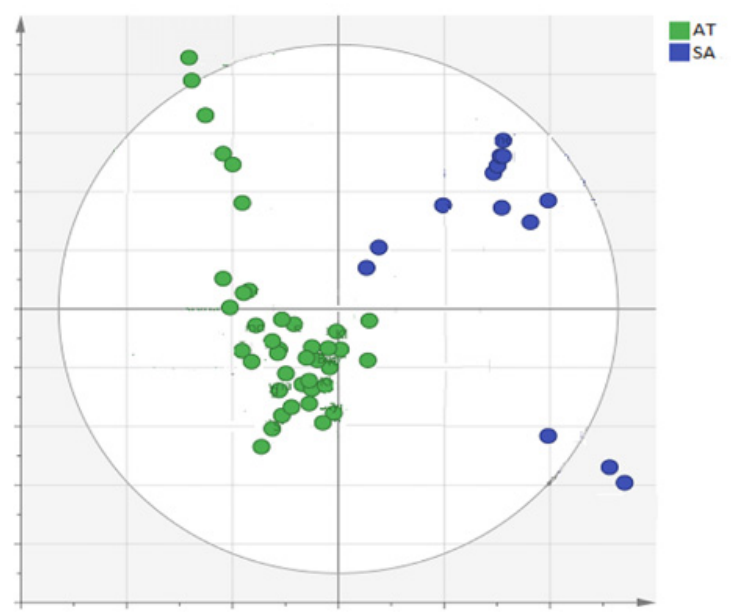

Figure 1. Biplots of the first and second principal components (PC) derived from PC analysis grape must sample obtained from different locations in Austria (AT) and South Africa (SA).

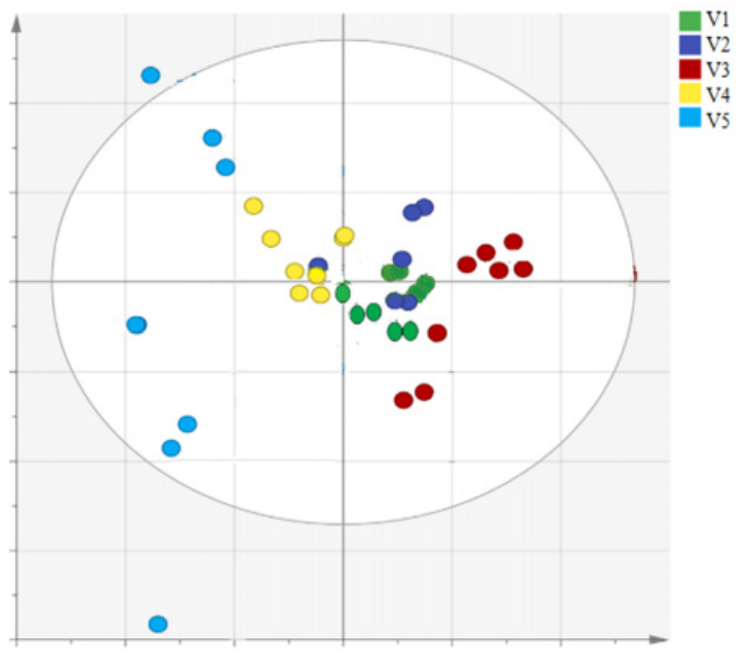

Figure 2. Biplots of the first and second principal components (PC) derived from PC analysis different grape varieties (V1-V5) obtained from different vineyards in Austria.

and characterization of regional-specific strains. However, it is important to keep in mind that the data are of a preliminary nature. While suggesting interesting differences in community profiles between countries, the data are based on samples collected during a single vintage, and confirmation of country-specific or grape variety-specific community profiles will require additional data sets from additional vintages. Future work will also focus on isolating indigenous strains from the South African and Austrian grape must in order to evaluate their contribution to the aromatic properties of wine.

\section{Literatur}

[1] International Organisation of Vine and Wine, Global economic vitiviniculture data (2017)

[2] Wesgro (Cape Town and Western Cape) Research report (July 2016) Sector: Wine. http:// wesgro.co.za/. Accessed, 18-09-2018

[3] SAWIS South African Wine Industry Statistics. vol. 42 (2017). http://www. sawis.o.za/ Accessed, 18-09-2018 
[4] A. Schnabl, S. Lappöhn, A. Pohl, Inst. Adv. Studies (2016)

[5] N. Bokulich, J. Thorngate, P. Richardson, D. Mills, Proc. Natl. Acad. Sci. USA 111, 139 (2014)

[6] V. Mezzasalma, A. Sandionigi, I. Bruni, A. Bruno, G. Lovicu, M. Casiraghi, PLoS ONE 12 (2017)

[7] C. Jara, E. Mateo, J.M. Guillamón, M.J. Torija, A. Mas, Int. J. Food Microbiol. 128, 336 (2008)
[8] E. Slabbert, C.J. Van Heerden, K. Jacobs, S. Afr. J. Sci. 106, 1 (2010)

[9] S.W. Culman, R. Bukowski, H.G. Gauch, H. Cadillo-Quiroz, D.H. Buckley, BMC Bioinf. 10, 171 (2009)

[10] Ø. Hammer, D.A.T. Harper, P.D. Ryan, Paleontologic (2001)

[11] K.R. Clarke, Aust. J. Ecol. 18, 117 (1993)

[12] C. Wang, D. García-Fernández, A. Mas, B. Esteve-Zarzoso, Front Microbiol. 6, 1 (2015) 